Cahiers $d u$ MONDE RUSSE

\section{Cahiers du monde russe}

Russie - Empire russe - Union soviétique et États indépendants

$59 / 4 \mid 2018$

Varia

\title{
Jérôme Bazin, Pascal Dubourg Glatigny, Piotr Piotrowski, eds., Art beyond Borders
}

Artistic Exchange in Communist Europe [1945-1989]

\section{Diana Plachendovskaya}

\section{(2) OpenEdition}

Journals

Édition électronique

URL : https://journals.openedition.org/monderusse/10907

DOI : 10.4000/monderusse. 10907

ISSN : $1777-5388$

Éditeur

Éditions de l'EHESS

\section{Édition imprimée}

Date de publication : 1 octobre 2018

Pagination : 718-721

ISBN : 978-2-7132-2747-9

ISSN : $1252-6576$

Référence électronique

Diana Plachendovskaya, « Jérôme Bazin, Pascal Dubourg Glatigny, Piotr Piotrowski, eds., Art beyond Borders ", Cahiers du monde russe [En ligne], 59/4 | 2018, mis en ligne le 01 octobre 2018, consulté le 08 janvier 2022. URL : http://journals.openedition.org/monderusse/10907 ; DOI : https://doi.org/ 10.4000/monderusse.10907 
Jérôme BAZIN, Pascal DUBOURG GLATIGNY, Piotr PIOTROWSKI, eds. Art beyond Borders

Artistic Exchange in Communist Europe [1945-1989]

Budapest - New York : CEU Press, 2016, 520 p.

Comment écrire l'histoire de l'art de l'Europe centrale et orientale sans délimiter ses frontières avec le reste de l'Europe et du monde ni la réduire à sa position régionale ? Une tentative audacieuse de réponse est fournie par l'ouvrage Art Beyond Borders : Artistic Exchange in Communist Europe [1945-1989], dans lequel les 36 contributeurs parviennent à démontrer que les questions que posent les œuvres et les pratiques artistiques de cette période sont non seulement communes aux pays dits du bloc de l'Est, mais aussi au reste de l'Europe. Le livre offre donc un panel d'études récentes sur les mouvements et les communications d'artistes et d'acteurs culturels de l'Europe centrale et orientale au-delà de son caractère régional. Dès l'introduction, les coordinateurs avertissent le lecteur de la porosité du rideau de fer malgré l'isolement de chaque pays du bloc et exposent leur ambition d'écrire une histoire de l'art transnationale. La diversité des profils des contributeurs de ce livre atteste que cette ambition a été prise au sérieux. Souvent originaires des pays de l'Europe de l'Est, ils sont aujourd'hui membres d'institutions éparpillées dans le monde entier. Leurs trajectoires professionnelles constituent un gage de leur connaissance de l'histoire de l'art qui s'est écrite en Occident et de la conscience qu'ils ont de la nécessité de sa réécriture.

Le livre est organisé autour de quatre axes thématiques qui déclinent les questions de l'échange et des circulations entre les frontières. La première partie, intitulée « Les personnes en mouvement », propose des textes consacrés aux déplacements temporaires ou permanents d'artistes en Europe et dans le monde. La 
contribution de Kai Artinger, par exemple, explore l'histoire des échanges entre le critique britannique John Berger et l'artiste soviétique Ernst Neizvestnij, qui a donné naissance au livre Art and Revolution : Ernst Neizvestny and The Role of the Artist in the URSS (1969). Il s'agit de la première monographie portant sur un artiste non officiel soviétique écrit par un influent intellectuel occidental. Outre le fait qu'il offrait une perspective sur la situation de l'art en URSS au lecteur extérieur, Berger assurait alors une certaine reconnaissance à Neizvestnij en Occident à un moment où l'artiste se trouvait isolé dans son propre pays. À côté de ces interactions insoupçonnées avec l'Europe occidentale, la production artistique est-européenne a pu aussi être le produit de trajectoires artistiques commencées sur le continent américain. Anja Jackes retrace ainsi la trajectoire du muralisme mexicain, genre artistique qui a franchi plusieurs fois dans son histoire les frontières européennes. Élaboré par David Alfaro Siqueiros et Diego Rivera au Mexique et en partie inspiré par les fresques de la Renaissance observées en Italie, le muralisme est importé en RDA par l'artiste espagnol immigré Josep Renau, qui travaillait alors à Halle-Neustadt sur un projet de décoration picturale de la nouvelle cité socialiste idéale.

Les essais de la deuxième partie, "Les objets en mouvement », prolongent la réflexion sur les circulations, sauf qu'ici l'attention se déplace vers des objets artistiques matériels ou immatériels (tels que la performance) et la mobilité des styles. Cette partie interroge les catégories habituelles des études sur l'art de l'Europe de l'Est : la tension entre le modernisme et le réalisme socialiste, les aspirations nationales ou internationales de la pratique artistique. Plus proche des méthodologies de l'histoire de l'art, cette partie se concentre davantage sur l'objet artistique et ses caractéristiques plastiques tout en restant en accord avec l'ambition principale de l'ouvrage qui consiste à démontrer la fragilité des frontières politiques et géographiques en matière d'échanges artistiques. Les auteurs repensent à partir des contextes locaux les mouvements connus du lecteur occidental comme le réalisme socialiste, l'art concret, Fluxus ou encore le mail art. À cela s'ajoutent les nouvelles catégories de l'art objectif de Leonhard Lapin ou de la propagarde de Max Herman (Maxy).

La partie « Rassembler les personnes » comporte des études sur les rassemblements officiels et non officiels d'artistes et d'intellectuels que sont les expositions, les festivals, les biennales, les conférences ou encore les réunions. Ces événements donnaient certes à voir la création artistique venue d'autres pays, mais ils permettaient surtout d'officialiser le processus de partage et de dialogue au sein du bloc. La dernière partie, « Définir l'Europe », boucle assez logiquement le « jeu d'échelles » (p. 17) recherché par les rédacteurs du volume. Cette section propose en effet de revoir la définition géographique de l'Europe au regard des liens que les artistes ont tissés avec d'autres pays socialistes comme la Chine, le Mexique, Cuba ou des pays de l'Asie centrale et de l'Afrique du Nord. Comme le suggèrent les directeurs du volume dans l'introduction, les frontières de l'Europe doivent être révisées d'abord du fait de l'obsolescence de la division du continent en deux parties soi-disant non communicantes. La séparation idéologique de l'époque semble avoir imprégné les différents domaines du savoir sur la région, y compris l'histoire de l'art. L'analyse 
des échanges fructueux avec le reste du monde permet d'élargir la perspective sur l'espace communiste de la période d'après-guerre au-delà de l'espace européen (ibid., p. 3).

Bien qu'elle offre des repères précieux pour le lecteur, la structuration en quatre parties pourrait paraître conventionnelle. En effet, ces parties sont peu problématisées autour des questions qui lient véritablement les contributions et l'activité artistique en Europe de l'Est en général. Elles sont certes mentionnées dans l'introduction : les tensions entre modernisme et réalisme socialiste, la géographie et les jeux d'échelles dans l'étude de l'histoire de l'art ainsi que la nécessité d'une nouvelle définition de l'Europe et de l'art européen incluant sa partie orientale. En ce sens, les propositions qui construisent cet ouvrage à partir de cas spécifiques, n'offrent pas de vision chronologique et synthétique de cette région. Ainsi, Art beyond Borders ne constitue pas une introduction à la matière, même si les articles pris séparément exposent avec clarté leurs cas d'études. En revanche, l'ouvrage propose de repenser les présupposés ancrés dans une histoire de l'art trop consensuelle, par exemple à l'égard du réalisme socialiste en tant que mouvement artistique imposé par Moscou exclusivement dans les pays de l'Est et utilisé à des fins de propagande. L'essai de Katarzyna Murawska-Muthesius « Remapping Socialist Realism : Renato Guttuso in Poland » revient ainsi sur l'histoire de l'adoption du style réaliste socialiste par plusieurs artistes occidentaux, démontrant ainsi l'adaptation de ce style à d'autres contextes en tant que pratique artistique engagée.

Comparé aux travaux existant sur l'art de l'Europe centrale et orientale, cet ouvrage se focalise davantage sur les communications à l'intérieur de la région et surtout vers l'extérieur. Les ouvrages majeurs comme East Art Map ${ }^{1}$ ou l'anthologie Primary Documents : A Sourcebook for Eastern and Central European Art Since the $1950 s^{2}$ perpétuent la délimitation du contexte est-européen afin de mieux circonscrire leur objet d'étude. En revanche, en remettant en question l'imperméabilité des frontières à l'intérieur du bloc de l'Est et avec le monde extérieur, Art Beyond Borders remet de fait en question l'existence de l'Europe de l'Est en tant que région.

L'ouvrage est le troisième volume de la série Leipzig Studies on the History and Culture of East-Central Europe. Cet ancrage de l'histoire de l'art au sein des études historiques et sociales montre non seulement l'importance de l'art en tant que champ d'influences politiques et indicateur des changements sociaux, mais aussi la diversité des méthodes ici utilisées. Néanmoins, l'ouvrage ne propose pas à proprement parler une nouvelle histoire de l'art qui mettrait l'étude des œuvres au premier plan.

Les nombreuses images en couleurs ou en noir et blanc accompagnent les textes plutôt qu'elles n'en constituent les objets d'études. Par ailleurs, une grande partie d'entre elles reproduisent des œuvres de commandes officielles, exécutées dans le style réaliste socialiste. L'attention particulière portée à l'art officiel témoigne du souci de renouveler l'étude de ce style artistique et de le dégager d'une perception de «non-art » ou de pratique indigne de l'avant-garde. Néanmoins, la séparation entre art non officiel et art officiel, qui a ensuite migré dans le discours de l'histoire de l'art, persiste. L'intérêt pour le réalisme socialiste est légitime et important aujourd'hui mais la manière d'intégrer cet art officiel dans l'histoire de l'art semble 
encore compliquée. Certains questionnements perdurent : faut-il apprécier au même niveau la production artistique officielle et la production non officielle issue d'un contexte totalitaire ? Peut-on faire abstraction de l'animosité du milieu alternatif de l'époque envers la culture officielle, de la différence de statuts sociaux des deux groupes d'artistes ainsi que des trajectoires de diffusion divergentes de leurs œuvres?

Les riches et diverses propositions scientifiques développées sur 520 pages fournissent des points de vue multiples sur les circulations artistiques et les pratiques de la production artistique en Europe de l'Est lors de la guerre froide. Cette fragmentation rend complexe la lecture du livre dans son intégralité et dans l'ordre proposé par les éditeurs. Cependant, elle est compatible avec l'ambition des éditeurs, qui, en rassemblant ces contributions, construisent à leur tour une cartographie de recherche comparable dans sa diversité à la cartographie des échanges artistiques à travers les frontières européennes de la période 1945-1989. L'ouvrage ne définit pas l'art du « bloc de l'Est », mais tente au contraire d'en déconstruire une définition trop figée, ce qui constitue une contribution importante à la réorientation de la recherche dans le domaine.

1 - IRWIN, ed., East Art Map : Contemporary Art and Eastern Europe, Londres : Afterall, 2006.

2 - Laura Hoptman, Tomáš Pospiszyl et alii, Primary Documents : A Sourcebook for Eastern and Central European Art since the 1950s, New York - Cambridge, MA London : The MIT Press, 2002.

\author{
Diana Plachendovskaya
}

CERCEC, CNRS, EHESS, PSL 